\title{
Dipyridine copper chloride as a mild and efficient catalyst for the solid state synthesis of 2-substituted benzimidazoles
}

\author{
J. Venu Madhav, B. Suresh Kuarm, and B. Rajitha* \\ Department of Chemistry, National Institute of Technology, Warangal 506 004, India \\ E-mail: rajitabhargavi@yahoo.com
}

\begin{abstract}
2-Substituted benzimidazoles have been synthesized in good yields from benzo[c][1,2,5]thiadiazole-4,5-diamine and aromatic aldehydes in the presence of catalytic amount of $\mathrm{CuPy}_{2} \mathrm{Cl}_{2}$, ground at room temperature.
\end{abstract}

Keywords: Benzimidazoles, benzo[c][1,2,5]thiadiazole-4,5-diamine, aromatic aldehyde, $\mathrm{CuPy}_{2} \mathrm{Cl}_{2}$, solvent free conditions

\section{Introduction}

Benzimidazole and its derivatives possess appreciable pharmacological activity such as anticancer, antimicrobial, pesticide, antihelmintic properties. ${ }^{1,2}$ Some derivatives have found commercial applications in several therapeutic areas, e.g., as anti-ulcerative, antiviral and antihistaminic agents. ${ }^{3,4}$ Benzimidazole derivatives also exhibit significant activity against several viruses such as HIV, ${ }^{5,6}$ herpes (HSV-1), ${ }^{7} \mathrm{RNA}^{8}$ influenza, ${ }^{9}$ and human cytomegalovirus (HCMV). ${ }^{5}$ Furthermore they have been used to act as topoisomerase inhibitors, ${ }^{10}$ selective neuropeptide YY1 receptor antagonists, ${ }^{11}$ angiotensin II inhibitors, ${ }^{12}$ potential antitumor agents 13 and smooth muscle cell proliferation inhibitors. ${ }^{14}$ In addition benzimidazoles are very important intermediates in organic synthesis, vitamin $\mathrm{B}_{12}$ constitutes a milestone in the chemistry of benzimidazoles.

As a result of their importance from a pharmacological, industrial and synthetic point of view, several methods for the preparation of benzimidazoles have been reported. These are the condensation of o-phenylenediamines and aldehydes in nitrobenzene, ${ }^{15}$ the condensation of oarylenediamines with carboxylic acids or their derivatives in the presence of strong acids such as polyphosphoric acid ${ }^{16}$ or mineral acids, ${ }^{17}$ boric acid ${ }^{18}$ and $p$-toluene sulphonic acid ( $p$-TSA) ${ }^{19}$ as catalyst. Direct condensation of o- arylenediamines and aldehydes is not a good synthetic reaction, as it is well known to yield a complex mixture-1, 2-disubstituted benzimidazoles, the bis-anil and dihydrobenzimidazoles as the main side products. ${ }^{20}$ In this case, however, the 
addition of transition metal, namely copper (II) acetate, ${ }^{21}$ lead tetraacetate ${ }^{22}$ allow a partial?? selective synthesis of benzimidazoles. Unfortunately, many of these methods have some drawbacks, such as drastic reaction conditions, low yields, co-occurrence of several side reactions, use of some toxic acids as catalyst, requirement of excess of reagents / catalyst and some reagents are expensive. Thus, the need for the development of an alternative route to construct the benzimidazole derivatives is in high demand.<smiles>Nc1ccc2nsnc2c1N</smiles>

1<smiles>[R]c1ccc(C=O)cc1</smiles>

2a-k

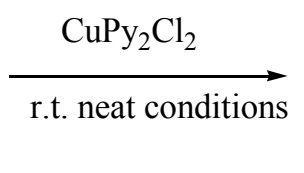<smiles>[R]c1ccc(-c2nc3c(ccc4nsnc43)[nH]2)cc1</smiles>

3a-k

\section{Scheme 1}

Table 1. $\mathrm{CuPy}_{2} \mathrm{Cl}_{2}$-Catalyzed formation of 7-aryl-6H-imidazo[4',5':3,4]benzo[1,2-c][1,2,5]thiadiazole

Entry


Table 1. Continued

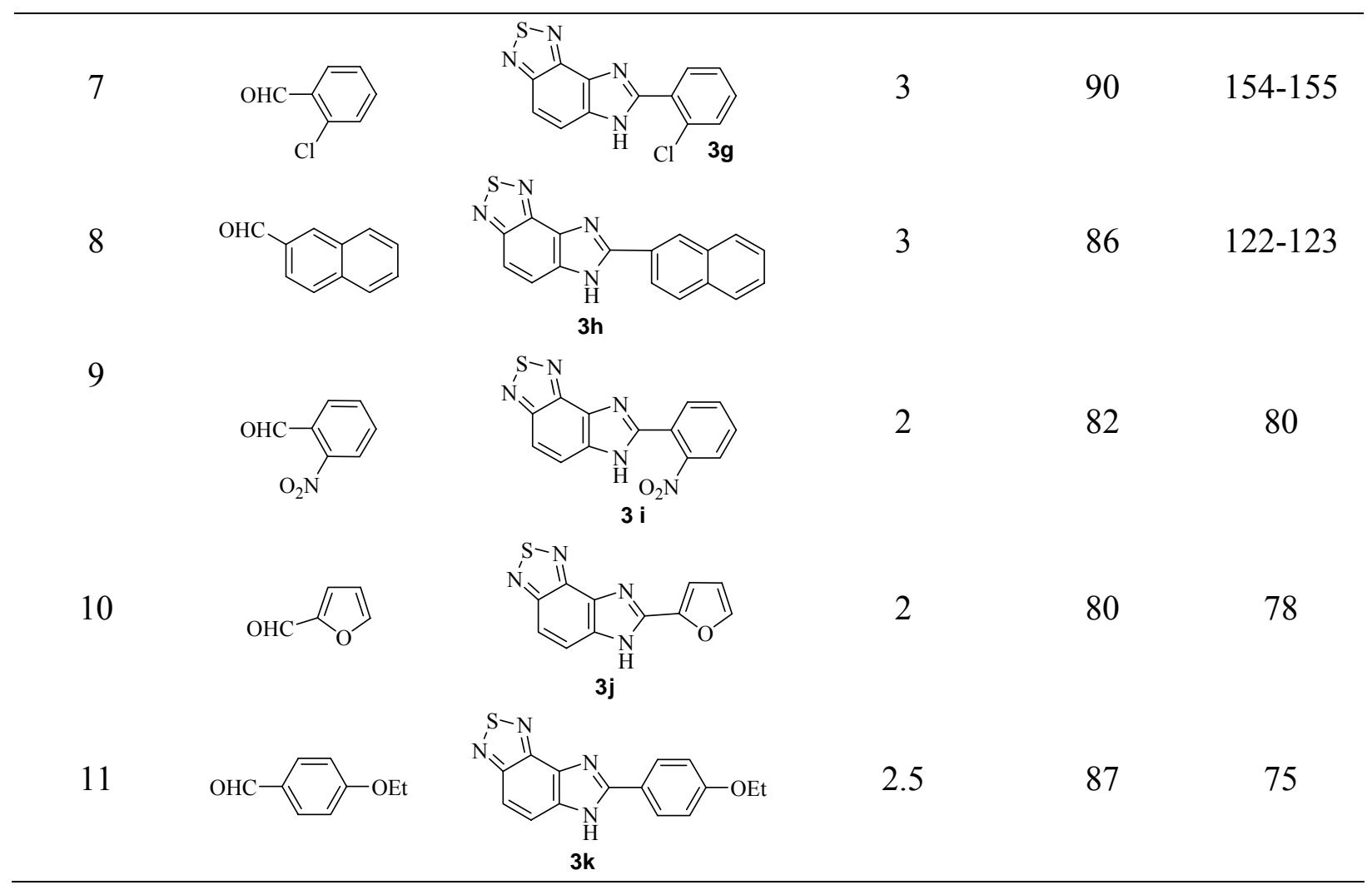

${ }^{\mathrm{a}}$ Yields refer to isolated pure products and were characterized by ${ }^{1} \mathrm{H}-\mathrm{NMR}$, IR and MS spectral data.

\section{Results and Discussion}

We elicited a new method for the synthesis of benzimidazoles with dipyridine copper chloride. Recently $\mathrm{CuPy}_{2} \mathrm{Cl}_{2}{ }^{23}$ has been used as Lewis acid catalyst. The catalyst is water tolerant, reusable and can effectively promote some organic reactions like Pechmann condensation ${ }^{24}$ and Biginelli reaction ${ }^{25}$ in good yields. In continuation of our work on this new catalyst, we wish to report in this paper the synthesis of 2-substituted benzimidazoles by the reaction between an aromatic aldehyde, benzo[c][1,2,5]thiadiazole-4,5-diamine ${ }^{26}$, and $\mathrm{CuPy}_{2} \mathrm{Cl}_{2}$ under solvent free conditions (Scheme 1). The reactions were carried out at room temperature, ground for 2-3 min using aldehyde $(3.6 \mathrm{~m} \mathrm{~mol})$ and diamine $(3 \mathrm{~m} \mathrm{~mol})$ in the presence of $\mathrm{CuPy}_{2} \mathrm{Cl}_{2}(0.01 \mathrm{mmol})$. The results are summarized in Table 1.

\section{Experimental Section}

General Procedures. Melting points were determined in open capillaries and are uncorrected. The reactions were monitored by TLC (thin layer chromatography) and visualized with UV light. 
IR spectra (KBr) were recorded on Shimadzu FTIR model 8010 spectrometer and the ${ }^{1} \mathrm{H}$ NMR spectra on Varian Gemini $200 \mathrm{MHz}$ spectrometer using TMS as an internal standard. Mass spectra were recorded on a JEOL JMS D-300 Spectrometer. The elemental analysis of the compounds was done on a Carlo Erba Model EA1108. All solvents and reagents were purchased from Aldrich and Fluka firms.

\section{Typical procedure}

To a mixture of 1, 2-diamine (3.0 m mol) and aldehyde $(3.6 \mathrm{~m} \mathrm{~mol})$ in a mortar, $\mathrm{CuPy}_{2} \mathrm{Cl}_{2}$ $(0.01 \mathrm{mmol})$ was added with continuous grinding with a pestle at room temperature. The progress of the reaction was monitored by TLC. After completion of the reaction, ethyl acetate $(10 \mathrm{ml})$ was added and the reaction mixture was washed with water $(6 \mathrm{ml})$. The organic layer was dried over anhydrous $\mathrm{Na}_{2} \mathrm{SO}_{4}$ and concentrated under vacuum to furnish the crude product, which was further purified by column chromatography (hexane/ethyl acetate $=8: 2$ ) and the product was characterized by spectroscopic data. All the reactions were completed within $3 \mathrm{~min}$. The aqueous layer containing the catalyst could be evaporated under reduced pressure $(50 \mathrm{~mm}$. $\mathrm{Hg}$ pressure at $85^{\circ} \mathrm{C}$ ) to give a blue solid. This could be reused for the next reaction with only modest loss in activity.

3a. IR $\left(\mathrm{KBr}, \mathrm{cm}^{-1}\right): 1619,3420 ;{ }^{1} \mathrm{H}$ NMR $\left(\mathrm{CDCl}_{3}\right): \delta=6.05(\mathrm{~s}, 1 \mathrm{H}), 7.05-7.85(\mathrm{~m}, 7 \mathrm{H})$; EIMS, 70Ev, $\mathrm{m} / \mathrm{z}: 251\left(\mathrm{M}^{+}\right)$; Anal.Calcd for $\mathrm{C}_{13} \mathrm{H}_{8} \mathrm{~N}_{4} \mathrm{~S}: \mathrm{C}, 61.89 ; \mathrm{H}, 3.20 ; \mathrm{N}, 22.21 ; \mathrm{S}, 12.71$; Found: $\mathrm{C}$, $61.87 ; \mathrm{H}, 3.24 ; \mathrm{N}, 22.23 ; \mathrm{S}, 12.74$.

3b. IR $\left(\mathrm{KBr}, \mathrm{cm}^{-1}\right): 1640,3350 ;{ }^{1} \mathrm{H}$ NMR $\left(\mathrm{CDCl}_{3}\right): \delta=5.80(\mathrm{~s}, 1 \mathrm{H}), 7.30-7.50(\mathrm{~m}, 4 \mathrm{H}), 7.70-$ 7.80(m, 2H); EIMS, 70Ev, m/z: $286\left(\mathrm{M}^{+}\right)$; Anal. Calcd for $\mathrm{C}_{13} \mathrm{H}_{7} \mathrm{ClN}_{4} \mathrm{~S}: \mathrm{C}, 54.45 ; \mathrm{H}, 2.46$; N, 19.54; S, 11.18; Found: C, 54.47; H, 2.45; N, 19.55; S, 11.20 .

3c. IR ( $\left.\mathrm{KBr}, \mathrm{cm}^{-1}\right): 1650,3385 ;{ }^{1} \mathrm{H}$ NMR $\left(\mathrm{CDCl}_{3}\right): \delta=6.20(\mathrm{~s}, 1 \mathrm{H})$ ), 7.60-8.05 (m, 6H); EIMS, 70Ev, m/z: $297\left(\mathrm{M}^{+}\right)$; Anal. Calcd for $\mathrm{C}_{13} \mathrm{H}_{7} \mathrm{~N}_{5} \mathrm{O}_{2} \mathrm{~S}: \mathrm{C}, 52.52 ; \mathrm{H}, 2.37$; N, 23.56; S, 10.79; Found: C, 52.54; H, 2.39; N, 23.52; S, 10.77 .

3d. IR $\left(\mathrm{KBr}, \mathrm{cm}^{-1}\right): 1640,3330 ;{ }^{1} \mathrm{H}$ NMR $\left(\mathrm{CDCl}_{3}\right): \delta=2.10(\mathrm{~s}, 3 \mathrm{H}), 4.50(\mathrm{~s}, 1 \mathrm{H}) 7.40-7.75(\mathrm{~m}$, 6H); EIMS, 70Ev, m/z: $266\left(\mathrm{M}^{+}\right)$; Anal. Calcd for $\mathrm{C}_{14} \mathrm{H}_{10} \mathrm{~N}_{4} \mathrm{~S}: \mathrm{C}, 63.14 ; \mathrm{H}, 3.78 ; \mathrm{N}, 21.04 ; \mathrm{S}$, 12.04; Found: C, 63.13; H, 3.76; N, 21.06; S, 12.07.

3e. IR $\left(\mathrm{KBr}, \mathrm{cm}^{-1}\right): 1635,3350 ;{ }^{1} \mathrm{H}$ NMR $\left(\mathrm{CDCl}_{3}\right): \delta=3.65(\mathrm{~s}, 3 \mathrm{H}), 5.50(\mathrm{~s}, 1 \mathrm{H}), 7.20-7.55(\mathrm{~m}$, 4H), 7.70-7.85(m, 2H); EIMS, 70Ev, m/z: $282\left(\mathrm{M}^{+}\right)$; Anal. Calcd for $\mathrm{C}_{14} \mathrm{H}_{10} \mathrm{~N}_{4} \mathrm{OS}$ : C, 59.56; $\mathrm{H}$, 3.57; N, 19.85; S, 11.36; Found: C, 59.59; H, 3.55; N, 19.87; S, 11.34 .

3f. IR $\left(\mathrm{KBr}, \mathrm{cm}^{-1}\right): 1640,3365 ;{ }^{1} \mathrm{H}$ NMR $\left(\mathrm{CDCl}_{3}\right): \delta=5.20(\mathrm{~s}, 1 \mathrm{H}), 7.10-7.55(\mathrm{~m}, 6 \mathrm{H}), 9.31(\mathrm{br} \mathrm{s}$, $1 \mathrm{H})$; EIMS, 70Ev, m/z: $268\left(\mathrm{M}^{+}\right)$; Anal. Calcd for $\mathrm{C}_{13} \mathrm{H}_{8} \mathrm{~N}_{4} \mathrm{OS}$ : C, 58.20; H, 3.01; N, 20.88; S, 11.95; Found: C, 58.23; H, 3.04; N, 20.87; S, 11.93.

3g. IR $\left(\mathrm{KBr}, \mathrm{cm}^{-1}\right): 1645,3350 ;{ }^{1} \mathrm{H}$ NMR $\left(\mathrm{CDCl}_{3}\right): \delta=5.82(\mathrm{~s}, 1 \mathrm{H}), 7.40-7.80(\mathrm{~m}, 6 \mathrm{H})$; EIMS, 70Ev, $m / z$ : $286\left(\mathrm{M}^{+}\right)$; Anal. Calcd for $\mathrm{C}_{13} \mathrm{H}_{7} \mathrm{ClN}_{4} \mathrm{~S}$ : C, 54.45; H, 2.46; N, 19.54; S, 11.18; Found: C, 54.48; H, 2.48; N, 19.57; S, 11.16 . 
3h. IR (KBr, $\left.\mathrm{cm}^{-1}\right): 1640,3365 ;{ }^{1} \mathrm{H}$ NMR $\left(\mathrm{CDCl}_{3}\right): \delta=6.10(\mathrm{~s}, 1 \mathrm{H})$ 7.45-7.90 (m, 9H); EIMS, 70Ev, m/z: $302\left(\mathrm{M}^{+}\right)$; Anal. Calcd for $\mathrm{C}_{17} \mathrm{H}_{10} \mathrm{~N}_{4} \mathrm{~S}: \mathrm{C}, 67.53 ; \mathrm{H}, 3.33 ; \mathrm{N}, 18.53$; S, 10.61; Found: C, 67.48; H, 3.41; N, 18.57; S, 10.58 .

3i. IR $\left(\mathrm{KBr}, \mathrm{cm}^{-1}\right): 1655,3425 ;{ }^{1} \mathrm{H}$ NMR $\left(\mathrm{CDCl}_{3}\right): \delta=6.35(\mathrm{~s}, 1 \mathrm{H}), 7.65-8.10(\mathrm{~m}, 6 \mathrm{H})$; EIMS, 70Ev, $\mathrm{m} / \mathrm{z}: 297\left(\mathrm{M}^{+}\right)$; Anal. Calcd for $\mathrm{C}_{13} \mathrm{H}_{7} \mathrm{~N}_{5} \mathrm{O}_{2} \mathrm{~S}: \mathrm{C}, 52.52 ; \mathrm{H}, 2.37$; N, 23.56; S, 10.79; Found: C, 52.56; H, 2.41; N, 23.52; S, 10.81 .

3j. IR (KBr, cm $\left.{ }^{-1}\right): 1640,3375 ;{ }^{1} \mathrm{H}$ NMR $\left(\mathrm{CDCl}_{3}\right): \delta=4.85(\mathrm{~s}, 1 \mathrm{H}), 6.95-7.21(\mathrm{~m}, 3 \mathrm{H}) ; 7.50-7.61$ (m, 2H); EIMS, 70Ev, m/z: $242\left(\mathrm{M}^{+}\right)$; Anal. Calcd for $\mathrm{C}_{11} \mathrm{H}_{6} \mathrm{~N}_{4} \mathrm{OS}: \mathrm{C}, 54.54 ; \mathrm{H}, 2.50$; N, 23.13; S, 13.24; Found: C, 54.56; H, 2.47; N, 23.18; S, 13.29 .

3k. IR $\left(\mathrm{KBr}, \mathrm{cm}^{-1}\right): 1640,3380 ;{ }^{1} \mathrm{H}$ NMR $\left(\mathrm{CDCl}_{3}\right): \delta=1.45(\mathrm{t}, 3 \mathrm{H}), 3.97(\mathrm{q}, 2 \mathrm{H}) ; 5.10(\mathrm{~s}, 1 \mathrm{H})$ 6.91-7.45 (m, 4H), 7.67-7.81 (m, 2H); EIMS, 70Ev, m/z: $296\left(\mathrm{M}^{+}\right)$; Anal. Calcd for $\mathrm{C}_{15} \mathrm{H}_{12} \mathrm{~N}_{4} \mathrm{OS}$ : C, 60.79; H, 4.08; N, 18.91; S, 10.82; Found: C, 60.81; H, 4.04; N, 18.96; S, 10.77.

\section{Conclusions}

In conclusion, we have described a simple and efficient method for the synthesis of 2- substituted benzimidazoles. The easy work-up procedure, non-toxic cost efficiency providing recyclability of the catalyst, excellent yields make this method a valid contribution to the existing methodologies.

\section{Acknowledgements}

Financial assistance from U.G.C (RGNF) Grant No: F.16-158/2006(SA-II) New Delhi is greatly acknowledged.

\section{References}

1. Tebbe, M. J.; Jensen, C. B.; Spitzer, W. A.; Franklin, R. B. Antiviral Res. 1999, 42, 25.

2. Sharma, S.; Abuzar, S. Prog. Drug. Res. 1983, 27, 85.

3. Spasov, A. A.; Yozhitsa, I. N.; Bugaeza, L. L.; Anisimova, V. A. Pharma. Chem. J. 1999, 33, 232.

4. Preston, P. N. The Chemistry of Heterocyclic compounds, Benzimidazoles and Congeneric Tricyclic Compounds; John Wiley\& Sons: New York, 1980; Vol. 40, Part 2; Ch. 10.

5. Porcari, A. R.; Devivar, R. V.; Kucera, L. S.; Drach, J. C.; Townsend, L. B. J. Med. Chem. 1998, 41, 1251.

6. Roth, M.; Morningstar, M. L.; Boyer, P. L.; Hughes, S. H.; Bukheit, R. W.; Michejda, C. J. J. Med. Chem. 1997, 40, 4199. 
7. Migawa, M. T.; Giradet, J. L.; Walker, J. A.; Koszalka, G. W.; Chamberlain, S. D.; Drach, J. C.; Townsend, L. B. J. Med. Chem. 1998, 41, 1242.

8. Tamm, I.; Seghal, P. B. Adv. Virus Res. 1978, 22, 187.

9. Tamm, I. Science 1957, 126, 1235.

10. Kim, J. S.; Gatto, B.; Yu, C.; Liu, A.; Liu, L. F.; Lavioe, E. J. Med. Chem. 1996, 39, 992.

11. Zarrinmayeh, H.; Zimmerman, D. M.; Cantrell, B. E.; Schober, D. A.; Bruns, R. F. Bioorg. Med. Chem. Lett. 1999, 9, 647.

12. Kohara, Y.; Kubo, K.; Imamiya, E.; Wada, T.; Inada, Y.; Naka, T. J. Med. Chem. 1996, 39, 5228.

13. Denny, W. A.; Rewcastle, G. W.; Bagley, B. C. J. Med. Chem. 1990, 33, 814.

14. Elokdah, H. M.; Chai, S. Y.; Sulkowski, T. S. US Patent, 1998, 5764 473; Chem. Abstr. 1998, 129, 58784g.

15. Sun, Q.; Yan, B. Bioorg. Med. Chem. Lett. 1998, 8, 361.

16. Preston, P. N. Benzimidazoles, and Congeneric Tricyclic Compounds, In Weissberger, A.; Taylor, E. C. Eds., Chemistry of Heterocyclic Compounds. Part 1, vol. 40, Wiley, New York, 1981, pp 6-60.

17. Grimmet, M. R. Imidazoles and their benzoderivatives, In Kartitzky, A. R.; Rees, C. W. Eds., Comprehensive Heterocyclic Chemistry, Vol. 5. Pergamon: Oxford, 1984, p. 457.

18. Pushkina, L. N.; Mazalov, S. A.; Poatovskii, I. Ya. Zh. Obshch. Khim. 1962, 32, 2624; Chem Abstr. 1963, 58, 9049h.

19. Yoshiyuki, T.; Kazuaki, Y. Hokkaido Daigaku Koagakubu Kenkyu Hokoku 1980. Chem Abstr. 1980, 93, 45-49204537k.

20. Smith, J. G.; Ho, I. Tetrahedron Lett. 1971, 38, 3541.

21. Weidenhagen, R. Ber. 1936, 69, 2263.

22. Stevens, F. F.; Bower, J. D. J. Chem. Soc. 1949, 2971.

23. Duntitz, J. D. Acta Cryst. 1957, 10, 307.

24. Rajitha, B.; Naveen Kumar, V.; Someshwar, P.; Venu Madhav, J.; Narsimha Reddy, P.; Thirupathi Reddy, Y. Arkivoc 2006, (xii), 23.

25. Naveen Kumar, V.; Someshwar, P.; Thirupathi Reddy, Y.; Narasimha Reddy, P.; Rajitha, B. J. Heterocycl. Chem. 2005, 42, 1017.

26. Komin, A. P.; Carmack, M. British Patent. 1968, 1152 814; J. Heterocycl. Chem. 1975, 14, 829. 